\title{
Poincaré theorem and nonlinear PDE's
}

\author{
by Maria E. Pliś (Kraków)
}

\begin{abstract}
A family of formal solutions of some type of nonlinear partial differential equations is found. Terms of such solutions are Laplace transforms of some Laplace distributions. The series of these distributions are locally finite.
\end{abstract}

1. Introduction. In this paper we consider the nonlinear partial differential equations of the form

$$
P(D) u=f(u)
$$

with $f$ analytic at zero, $f(0)=0$, and $f^{\prime}(0) \neq 0$. This means that $f(u)=$ $\sum_{m=1}^{\infty} c_{m} u^{m}$ with $c_{1} \neq 0$. The operator $P(D)$ is a linear differential operator $P(\partial / \partial x)$, where $P(z)=P\left(z_{1}, \ldots, z_{n}\right)$ is a complex polynomial without constant term.

Our aim is to find the behaviour of a solution of the equation (1) at infinity, writing this solution as some series of integrals involving a solution of the linear part of (1), i.e.

$$
P(D) y=c_{1} y
$$

The method of construction used here follows Bobylev [2] (see also Rosales [3]). They solve only evolution type nonlinear equations ( $\mathrm{KdV}$, sineGordon etc.) in this way. Applying essentially Bobylev's idea to use the Poincaré theorem on normal forms (see Arnold [1]) we can solve a wide enough class of equations (1). The same method can be used in the case of the right hand side of (1) being a function not only of $u$ but also of the derivatives of $u$.

1991 Mathematics Subject Classification: 44A10, 46F20.

Key words and phrases: Laplace distributions, Laplace transforms, formal solutions. Supported by KBN grant 2-P03A-006-08. 
2. Construction of formal solutions. We can write (1) as

$$
P(D) u-c_{1} u=\sum_{m=2}^{\infty} c_{m} u^{m}
$$

Let $Z=\left\{\left(z_{1}, \ldots, z_{n}\right) \in \mathbb{C}^{n}: P(z)-c_{1}=0\right\}$ and assume that $Z$ contains a curve $Z^{\prime} \subset Z$ described by

$$
Z^{\prime}=\left\{z=\left(z_{1}, \ldots, z_{n}\right) \in Z: z=z(k)=\left(z_{1}(k), \ldots, z_{n}(k)\right), k \in \mathbb{R}\right\},
$$

such that $\left(Z^{\prime}+Z^{\prime}\right) \cap Z^{\prime}=\emptyset$. Let $y=y\left(x_{1}, \ldots, x_{n}\right)$ be a solution of the linear equation $(2)$ given by the integral over $Z^{\prime}$ :

$$
y\left(x_{1}, \ldots, x_{n}\right)=\int_{-\infty}^{\infty} e^{-z_{1}(k) x_{1}-\ldots-z_{n}(k) x_{n}} \Phi(k) d k
$$

with $\Phi$ being an arbitrary function such that the integral (3) makes sense for $x=\left(x_{1}, \ldots, x_{n}\right)$ large enough.

We use the following notations. Let $L u=\frac{1}{c_{1}} P(D) u$. We denote by $H_{m}$ for $m \geq 2$ the special $m$-linear form

$$
H_{m}\left[u_{1}, \ldots, u_{m}\right]=\frac{c_{m}}{c_{1}} u_{1}(0) \ldots u_{m}(0) .
$$

Let $u^{x}(t)=u(x+t), x, t \in \mathbb{C}^{n}$, and let $Q\left(u_{1}, \ldots, u_{m}\right)$ denote the function $x \mapsto Q\left[u_{1}^{x}, \ldots, u_{m}^{x}\right], Q$ being an $m$-linear form.

Thus (1) and (2) can be written as

$$
u=L u-\sum_{m=2}^{\infty} H_{m}(u, \ldots, u)
$$

and

$$
y=L y .
$$

We are looking for a solution $u$ represented by a formal series

$$
u=y+\sum_{j=2}^{\infty} R_{j}(y, \ldots, y)
$$

with some $j$-linear form $R_{j}$. From (6) we get

$$
\begin{aligned}
y & =u-\sum_{j=2}^{\infty} R_{j}(y, \ldots, y) \\
& =u-R_{2}(u, u)+\sum_{m=3}^{\infty} \sum_{j=2}^{m} \sum_{|p|=m, p_{k} \geq 1}(-1)^{s_{j}+1} R_{j}\left(R_{p_{1}}, \ldots, R_{p_{j}}\right),
\end{aligned}
$$

with the convention $R_{1}(u)=u,|p|=p_{1}+\ldots+p_{j}$ and $s_{j}=\#\left\{k: p_{k}>1\right\}$. 
Thus

(8)

$$
\begin{aligned}
L y= & L u-L R_{2}(u, u) \\
& -\sum_{m=3}^{\infty} \sum_{j=2}^{m} \sum_{|p|=m, p_{k} \geq 1}(-1)^{s_{j}+1} L R_{j}\left(R_{p_{1}}, \ldots, R_{p_{j}}\right) .
\end{aligned}
$$

From (6) and (5) we get

$u=L y$

$$
+\sum_{j=2}^{\infty} \frac{1}{j}\left\{R_{j}(L y, y, \ldots, y)+R_{j}(y, L y, \ldots, y)+\ldots+R_{j}(y, \ldots, y, L y)\right\} .
$$

Now by (7) and (8) we have

$$
\begin{aligned}
u= & L u-L R_{2}(u, u)-\sum_{m=3}^{\infty} \sum_{j=2}^{m} \sum_{|p|=m}(-1)^{s_{j}+1} L R_{j}\left(R_{p_{1}}, \ldots, R_{p_{j}}\right) \\
& +\sum_{j=2}^{\infty} \frac{1}{j}\left\{R_{j}\left(L u-L R_{2}(u, u)-\ldots, \ldots, u-R_{2}(u, u)-\ldots\right)+\ldots\right. \\
& \left.+R_{j}\left(u-R_{2}(u, u) \ldots, \ldots, L u-L R_{2}(u, u) \ldots\right)\right\} \\
= & L u-\sum_{m=2}^{\infty} \sum_{j=2}^{m} \sum_{|p|=m}(-1)^{s_{j}+1}\left\{L R_{j}\left(R_{p_{1}}, \ldots, R_{p_{j}}\right)\right. \\
& \left.-\frac{1}{j}\left(R_{j}\left(L R_{p_{1}}, \ldots, R_{p_{j}}\right)+\ldots+R_{j}\left(R_{p_{1}}, \ldots, L R_{p_{j}}\right)\right)\right\} .
\end{aligned}
$$

Now we compare the $m$ th order terms and obtain a recurrence system of equations for $R_{m}, m \geq 2$ :

$$
\begin{aligned}
H_{m}= & \sum_{j=2}^{m} \sum_{|p|=m}(-1)^{s_{j}+1}\left\{L R_{j}\left(R_{p_{1}}, \ldots, R_{p_{j}}\right)\right. \\
& \left.-\frac{1}{j}\left(R_{j}\left(L R_{p_{1}}, \ldots, R_{p_{j}}\right)+\ldots+R_{j}\left(R_{p_{1}}, \ldots, L R_{p_{j}}\right)\right)\right\} .
\end{aligned}
$$

To find $R_{m}$ we use the Laplace transformation. To this end set $e_{q}(x)=$ $e^{-q x}$ (so, according to our previous notation, $e_{q}^{0}(\tau)=e^{-q \tau}$ ), and

$$
\begin{aligned}
\lambda(q) & =L\left[e_{q}^{0}\right]\left(=L\left(e_{q}\right)(0)\right), \\
h_{m}\left(q_{1}, \ldots, q_{m}\right) & =H_{m}\left[e_{q_{1}}^{0}, \ldots, e_{q_{m}}^{0}\right]=\frac{c_{m}}{c_{1}}, \\
r_{m}\left(q_{1}, \ldots, q_{m}\right) & =R_{m}\left[e_{q_{1}}^{0}, \ldots, e_{q_{m}}^{0}\right] .
\end{aligned}
$$

Here obviously $q, q_{j}, x, \tau \in \mathbb{C}^{n}$ for $j=1, \ldots, m$, therefore $q x$ means the scalar product. 
Applying (9) to the system $\left[e_{q_{1}}^{0}, \ldots, e_{q_{m}}^{0}\right]$ we get, for $m=2$,

$$
h_{2}\left(q_{1}, q_{2}\right)=r_{2}\left(q_{1}, q_{2}\right)\left[\lambda\left(q_{1}+q_{2}\right)-\frac{1}{2}\left(\lambda\left(q_{1}\right)+\lambda\left(q_{2}\right)\right)\right],
$$

and for $m>2$, defining $p_{l}^{\prime}=p_{1}+\ldots+p_{l}(l=1, \ldots, j)$,

$$
\begin{aligned}
h_{m}\left(q_{1}, \ldots, q_{m}\right) & \\
= & \sum_{j=2}^{m} \sum_{|p|=m}(-1)^{s_{j}+1} r_{j}\left(q_{1}+\ldots+q_{p_{1}}, \ldots, q_{p_{j-1}^{\prime}+1}+\ldots+q_{m}\right) \\
& \times r_{p_{1}}\left(q_{1}, \ldots, q_{p_{1}}\right) \ldots r_{p_{j}}\left(q_{p_{j-1}^{\prime}+1}, \ldots, q_{m}\right) \\
& \times\left[\lambda\left(q_{1}+\ldots+q_{m}\right)\right. \\
& \left.\quad-\frac{1}{j}\left(\lambda\left(q_{1}+\ldots+q_{p_{1}}\right)+\ldots+\lambda\left(q_{p_{j-1}^{\prime}+1}+\ldots+q_{m}\right)\right)\right] .
\end{aligned}
$$

Therefore if $\Delta_{j}\left(t_{1}, \ldots, t_{j}\right)=\lambda\left(t_{1}+\ldots+t_{j}\right)-\frac{1}{j}\left[\lambda\left(t_{1}\right)+\ldots+\lambda\left(t_{j}\right)\right]$ then we have the recurrence system of formulas for $r_{m}$, with $q=\left(q_{1}, \ldots, q_{m}\right)$ :

(10)

$$
\left\{\begin{array}{l}
r_{2}(q) \Delta_{2}(q)=c_{2} / c_{1}, \\
r_{m}(q) \Delta_{m}(q)=c_{m} / c_{1}+\ldots \\
\quad+\sum_{j=2}^{m-1} \sum_{|p|=m}(-1)^{s_{j}+1} r_{j}\left(q_{1}+\ldots+q_{p_{1}}, \ldots, q_{p_{j-1}^{\prime}+1}+\ldots+q_{m}\right) \\
\quad \times r_{p_{1}}\left(q_{1}, \ldots, q_{p_{1}}\right) \ldots r_{p_{j}}\left(q_{p_{j-1}^{\prime}+1}, \ldots, q_{m}\right) \\
\quad \times \Delta_{j}\left(q_{1}+\ldots+q_{p_{1}}, \ldots, q_{p_{j-1}^{\prime}+1}+\ldots+q_{m}\right) .
\end{array}\right.
$$

Now, with $y$ given by (3), we can write

$$
\begin{aligned}
R_{m}(y, \ldots, y)(x) & \\
= & R_{m}\left[\int_{-\infty}^{\infty} e^{-z\left(k_{1}\right)\left(x+\tau_{1}\right)} \Phi\left(k_{1}\right) d k_{1}, \ldots, \int_{-\infty}^{\infty} e^{-z\left(k_{m}\right)\left(x+\tau_{m}\right)} \Phi\left(k_{m}\right) d k_{m}\right] \\
& =\int_{\mathbb{R}^{m}} e^{-\left[z\left(k_{1}\right)+\ldots+z\left(k_{m}\right)\right] x} \Phi\left(k_{1}\right) \ldots \Phi\left(k_{m}\right) R_{m}\left(e_{z(k)}(0)\right) d k_{1} \ldots d k_{m} \\
& =\int_{\mathbb{R}^{m}} r_{m}(z(k)) e^{-\left[z\left(k_{1}\right)+\ldots+z\left(k_{m}\right)\right] x} \Phi\left(k_{1}\right) \ldots \Phi\left(k_{m}\right) d k .
\end{aligned}
$$

Thus we have proved

Theorem. Assume that $\left|\Delta_{j}\left(t_{1}, \ldots, t_{j}\right)\right| \geq A>0$ on the set

$$
W_{j}\left(p_{1}, \ldots, p_{j}\right)=\left\{\left(t_{1}, \ldots, t_{j}\right): t_{k} \in p_{k} Z^{\prime}, k=1, \ldots, j\right\}
$$


for every $j \in \mathbb{N}, j \geq 2$ and every $\left(p_{1}, \ldots, p_{j}\right) \in \mathbb{N}^{j}$. Then we have a solution $u$ of (1) given by a formal series

$$
u(x)=\sum_{m=1}^{\infty} \int_{\mathbb{R}^{m}} r_{m}(z(k)) \Phi\left(k_{1}\right) \ldots \Phi\left(k_{m}\right) e^{-\left[z\left(k_{1}\right)+\ldots+z\left(k_{m}\right)\right] x} d k
$$

with $r_{m}$ defined by (10) and $\Phi$ as in (3).

3. Example. Consider the equation

$$
\Delta u=\frac{\partial^{2} u}{\partial x_{1}^{2}}+\frac{\partial^{2} u}{\partial x_{2}^{2}}=\sum_{j=1}^{\infty} c_{j} u^{j} .
$$

We can write it as

$$
\Delta u-a u=b u+\sum_{j=2}^{\infty} c_{j} u^{j},
$$

with $a+b=c_{1}, a>0$. Then $P(D)=\Delta-a$ and $\lambda(q)=q_{1}^{2}+q_{2}^{2}-a$. We choose $Z^{\prime}=\left\{\left(i k, \sqrt{a+k^{2}}\right): k \in \mathbb{R}\right\}$ and we can see that $Z^{\prime}+\ldots+Z^{\prime}=n Z^{\prime}=$ $\left\{(i x, y): y \geq \sqrt{n^{2} a+x^{2}}, x, y \in \mathbb{R}\right\} \subset \mathbb{C}^{2}, 2 Z^{\prime} \cap Z^{\prime}=\emptyset$ and $n Z^{\prime} \subset(n-1) Z^{\prime}$. Moreover, $\bigcup_{n=2}^{\infty}\left(\mathbb{C}^{2} \backslash n Z^{\prime}\right)=\mathbb{C}^{2}$.

We have

$$
\begin{aligned}
& \lambda\left(z\left(k_{1}\right)+\ldots+z\left(k_{l}\right)\right) \\
& \quad=\left(i\left(k_{1}+\ldots+k_{l}\right)\right)^{2}+\left(\sqrt{a+k_{1}^{2}}+\ldots+\sqrt{a+k_{l}^{2}}\right)^{2}-a \\
& \quad=(l-1) a+\sum_{1 \leq m<j \leq l} 2\left(\sqrt{a+k_{m}^{2}} \sqrt{a+k_{j}^{2}}-k_{m} k_{j}\right) \geq\left(l^{2}-1\right) a>0 .
\end{aligned}
$$

For $t_{l} \in p_{l} Z^{\prime}(l=1, \ldots, r)$ and $m=p_{1}+\ldots+p_{r}(r \geq 2)$ we also have $\Delta_{r}\left(t_{1}, \ldots, t_{r}\right)$

$$
\begin{aligned}
= & \Delta_{r}\left(z\left(k_{1}\right)+\ldots+z\left(k_{p_{1}}\right), \ldots, z\left(k_{p_{r-1}^{\prime}}+1\right)+\ldots+z\left(k_{m}\right)\right) \\
= & \lambda\left(z\left(k_{1}\right)+\ldots+z\left(k_{m}\right)\right)-\frac{1}{r}\left[\lambda\left(z\left(k_{1}\right)+\ldots+z\left(k_{p_{1}}\right)\right)+\ldots\right. \\
& \left.+\lambda\left(z\left(k_{p_{r-1}^{\prime}+1}\right)+\ldots+z\left(k_{m}\right)\right)\right] \\
\geq & {\left[\left(1-\frac{1}{r}\right) m^{2}+2\left(1-\frac{1}{r}\right)\right] a=\frac{r-1}{r}\left(m^{2}+2\right) a>0 . }
\end{aligned}
$$

So the assumptions of the Theorem are satisfied.

4. Locally finite representation of formal solutions. Assume now that there exists a linear map $A: \mathbb{C}^{n} \rightarrow \mathbb{C}^{n}$ such that $A\left(Z^{\prime}\right) \subset \mathbb{R}_{+}^{n}$. We define $\widehat{\mathbb{R}}_{+}^{n}=A^{-1}\left(\mathbb{R}_{+}^{n}\right)$. We consider the space $L_{(\omega)}^{\prime}\left(\mathbb{R}_{+}^{n}\right)$ of Laplace distributions 
(of type $\omega \in \mathbb{R}^{n}$, see [4] or [5]), supported by $\widehat{\mathbb{R}}_{+}^{n}$, and denote by $L_{(\omega)}\left(\widehat{\mathbb{R}}_{+}^{n}\right)$ the space of test functions $\phi=\psi \circ A$ for some $\psi \in L_{(\omega)}\left(\mathbb{R}_{+}^{n}\right)$.

Consider a functional $S_{1}$ defined for $\phi \in L_{(\omega)}\left(\widehat{\mathbb{R}}_{+}^{n}\right)$ by

$$
S_{1}[\phi]=\int_{-\infty}^{\infty} \phi(z(k)) \Phi(k) d k
$$

with $\Phi$ as in (3). Obviously $y$ in (3) is the value of $S_{1}$ on $\phi(z)=e^{-z x}$. Now we assign to $S_{1}$ a defining function $\Psi_{1}$ :

$$
\Psi_{1}(z)=\frac{1}{(2 \pi i)^{n}} S_{1}\left[\frac{e^{-(z-w)^{2}}}{(z-w)^{1}}\right]
$$

for $z \in \mathbb{C}^{n} \# \widehat{\mathbb{R}}_{+}^{n}:=A^{-1}\left(\mathbb{C}^{n} \# \mathbb{R}_{+}^{n}\right)$, where by $\mathbb{C}^{n} \# \mathbb{R}_{+}^{n}$ we understand $\left(\mathbb{C} \backslash \mathbb{R}_{+}\right)^{n}$. We can see by $(13)$ that

$$
\Psi_{1}(z)=\frac{1}{(2 \pi i)^{n}} \int_{-\infty}^{\infty} \frac{e^{-(z-z(k))^{2}}}{(z-z(k))^{\mathbf{1}}} \Phi(k) d k .
$$

Therefore, for $\phi \in L_{(\omega)}\left(\widehat{\mathbb{R}}_{+}^{n}\right)$, we can write (cf. [5])

$$
S_{1}[\phi]=\sum_{\sigma \in\{-1,1\}^{n}} \operatorname{sgn} \sigma \lim _{\varepsilon \rightarrow 0^{+}} \int_{\widehat{\mathbb{R}}_{+}^{n}} \phi(u) \Psi_{1}(u+i \sigma \varepsilon) d u .
$$

Hence for the solution $y$ of (2) we get the following formula:

$$
\begin{aligned}
y(x) & =S_{1}\left[e^{-z x}\right] \\
& =\sum_{\sigma \in\{-1,1\}^{n}} \operatorname{sgn} \sigma \lim _{\varepsilon \rightarrow 0^{+}} \frac{1}{(2 \pi i)^{n}} \int_{\widehat{\mathbb{R}}_{+}^{n}} \int_{-\infty}^{\infty} \frac{e^{-z x-(z+i \sigma \varepsilon-z(k))^{2}}}{(z+i \sigma \varepsilon-z(k))^{\mathbf{1}}} \Phi(k) d k d z .
\end{aligned}
$$

For $m \geq 2$, the $m$ th term of the formal solution $u$ is the value on $\phi(z)=$ $e^{-z x}$ of the Laplace distribution $S_{m}$ given by

$$
S_{m}[\phi]=\int_{\mathbb{R}^{m}} \phi\left(z\left(k_{1}\right)+\ldots+z\left(k_{m}\right)\right) \Phi\left(k_{1}\right) \ldots \Phi\left(k_{m}\right) r_{m}(z(k)) d k
$$

with $r_{m}$ given by (10). By analogy with the case $m=1$, we obtain the defining function for $S_{m}$ :

$$
\begin{aligned}
\Psi_{m}(z) & =\frac{1}{(2 \pi i)^{n}} S_{m}\left[\frac{e^{-(z-w)^{2}}}{(z-w)^{\mathbf{1}}}\right] \\
& =\frac{1}{(2 \pi i)^{n}} \int_{\mathbb{R}^{m}} \frac{e^{-\left(z-\left(z\left(k_{1}\right)+\ldots+z\left(k_{m}\right)\right)\right)^{2}}}{\left(z-\left(z\left(k_{1}\right)+\ldots+z\left(k_{m}\right)\right)\right)^{\mathbf{1}}} \Phi\left(k_{1}\right) \ldots \Phi\left(k_{m}\right) r_{m}(z(k)) d k .
\end{aligned}
$$


Hence we get

$$
S_{m}\left[e^{-z x}\right]=\sum_{\sigma \in\{-1,1\}^{n}} \operatorname{sgn} \sigma \lim _{\varepsilon \rightarrow 0^{+}} \int_{\widehat{\mathbb{R}}_{+}^{n}} e^{-z x} \Psi_{m}(z+i \sigma \varepsilon) d z .
$$

We see that for $m \geq 2, \operatorname{supp} S_{m}=m Z^{\prime}$, and $R_{m}(y, \ldots, y)(x)=S_{m}\left[e^{-z x}\right]$. So if $(m+1) Z^{\prime} \subset m Z^{\prime}$, then we have

$$
u(x)=\sum_{m=1}^{\infty} S_{m}\left[e^{-z x}\right]=S\left[e^{-z x}\right]
$$

and this formal series is locally finite, that is, for every $\phi \in L_{(\omega)}\left(\widehat{\mathbb{R}}_{+}^{n}\right)$ with $\operatorname{supp} \phi$ bounded, $S[\phi]$ is the sum of a finite number of terms. More precisely, let $\phi(x)=0$ for $|x| \geq M, x \in \widehat{\mathbb{R}}_{+}^{n}$. Since we can find $N \in \mathbb{N}$ such that $N Z^{\prime} \cap\{x:|x|<M\}=\emptyset$ we have $S_{m}[\phi]=0$ for $m \geq N$. So $S[\phi]=$ $\sum_{m=1}^{N-1} S_{m}[\phi]$.

\section{References}

[1] V. I. Arnold, Additional Topics in the Theory of Ordinary Differential Equations, Nauka, Moscow, 1978 (in Russian).

[2] A. Bobylev, Poincaré theorem, Boltzmann equation and KdV-type equations, Dokl. Akad. Nauk SSSR 256 (1981), 1341-1346 (in Russian).

[3] R. R. Rosales, Exact solutions of some nonlinear evolution equations, Stud. Appl. Math. 59 (1978), 117-151.

[4] Z. Szmydt and B. Ziemian, Laplace distributions and hyperfunctions on $\overline{\mathbb{R}}_{+}^{n}$, J. Math. Sci. Tokyo 5 (1998), 41-74.

[5] B. Ziemian, Generalized analytic functions with applications to singular ordinary and partial differential equations, Dissertationes Math. 354 (1996).

Institute of Mathematics

Cracow Pedagogical University

Podchorążych 2

30-084 Kraków, Poland

E-mail: meplis@wsp.krakow.pl 